\title{
Application and Mechanism Analysis of Tongdu Liyan Acupuncture in Treating Dysphagia in Parkinson's Disease
}

\author{
Miaolin Yang ${ }^{1}$, Weixing Feng ${ }^{2, *}$, Binfeng $\mathrm{Li}^{2}$ \\ ${ }^{1}$ School of Acupuncture and Tuina, Shaanxi University of Chinese Medicine, Xianyang 712046, China. \\ ${ }^{2}$ Affiliated Hospital of Shaanxi University of Chinese Medicine, Xianyang 712000, China.
}

\begin{abstract}
How to cite this paper: Miaolin Yang, Weixing Feng, Binfeng Li. (2021) Application and Mechanism Analysis of Tongdu Liyan Acupuncture in Treating Dysphagia in Parkinson's Disease. International Journal of Clinical and Experimental Medicine Research, 5(3), 304-309.

DOI: 10.26855/ijcemr.2021.07.012
\end{abstract}

Received: April 7, 2021

Accepted: May 12, 2021

Published: June 15, 2021

*Corresponding author: Weixing Feng, Affiliated Hospital of Shaanxi University of Chinese Medicine, Xianyang 712000, China.

Email: 2395613596@qq.com

\begin{abstract}
Parkinson's disease is located in the brain, and the direct circulation and indirect circulation of the governor meridian as well as the physiological function of the Governor Meridian are closely related to the brain and swallowing. Weixing Feng, deputy chief physician, believes that the etiology and pathogenesis of Parkinson's disease dysphagia were brain collateral damage, obstruction of the governor vessel, and the loss of degenerative mechanism. “Tongdu Liyan” acupuncture is Weixing Feng's experience group of acupoints and effective methods in the treatment of deglutition disorders in Parkinson's disease. "Tongdu Li" is an important idea in the treatment of Parkinson's disease to cure the disease, while "Liyan" is an effective therapy for deglutition disorders to treat both the symptoms and root causes. This paper will expatiate on the acupoint group, therapeutic principle, operation method, contraindications, mechanism of action and clinical trials of to treat the disease.
\end{abstract}

\section{Keywords}

Parkinson's Disease, Dysphagia, Tongdu Liyan Acupuncture Method, Governor Vessel

Parkinson's disease (PD) is a chronic degenerative disease of the nervous system. The main clinical manifestations of the disease are mainly motor symptoms, and non-motor symptoms cannot be ignored. Among them, dysphagia is one of the common non-motor symptoms. According to statistics, the incidence of PD dysphagia is as high as $82 \%$ [1], which increases the chance of aspiration and lung infection in patients, and seriously threatens the health and quality of life of patients [2]. At present, Western medicine is still inconclusive on the pathogenesis of PD swallowing dysfunction. Oral drugs (levodopa preparations) are difficult to treat, and the effectiveness of the drugs is not accurate. In recent years, doctors have paid more and more attention to clinical research on the treatment of PD dysphagia with acupuncture, and its efficacy has been gradually affirmed. Instructor Weixing Feng is the deputy chief physician of the Affiliated Hospital of Shaanxi University of Chinese Medicine. In the clinical treatment of Parkinson's disease dysphagia, he discovered and summarized a set of effective acupuncture methods and acupoints for the treatment of PD dysphagia- “Tongdu Liyan” Stitch. In the process of following, the clinical practice of the instructor, the author found that the "Tongdu Liyan" acupuncture method used to treat PD dysphagia has fewer acupoints, and is simple and effective. The report is as follows. 


\section{The acupoint group of "Tongdu Liyan” acupuncture method}

The "Tongdu Liyan" acupuncture method is proposed in accordance with the principle of local acupoint selection combined with disease differentiation. The specific acupoint selection are: Three needles in the pharynx, Lianquan, Shenting, Yintang, Baihui, Fengfu, and Naohu.

Teacher Weixing Feng has been engaged in clinical acupuncture and moxibustion for more than 20 years. He has been devoted to the research of dysphagia, especially the dysphagia caused by cerebrovascular disease. In the treatment of stroke dysphagia, the instructor put forward the experience of choosing acupuncture points "three needles" in the continuous exploration, namely the back wall of the pharynx, the tip of the tongue, and Jinjin and Yuye. After many verifications, it has been found that its clinical efficacy is positive [3-4]. In recent years, in the treatment of dysphagia associated with PD, the instructor still chooses the "three pharynx" as the main acupoint, which has a certain clinical effect [5]. As PD is a degenerative disease, the lesion is in the brain, and the Du Channel is closely related to the brain in circulation, physiology, and pathology. Therefore, the instructor will select the Governor Meridian acupoints and the "three needles in the pharynx" during acupuncture to enhance the therapeutic effect [6].

\section{Principles of “Tongdu Liyan” acupuncture method}

In the implementation of the "Tongdu Liyan" acupuncture method, the principles of replenishing the deficiency and reducing the solid, adapting measures to individual conditions, and seeking the root of the disease are the principles. When acupuncturing the Governor Meridian acupoints, the methods of replenishing and reducing are different. The specific operation of some acupoints should follow the treatment method tailored to the individual's conditions: Combining the patient's tongue coating and pulse condition, adopt the method of supplementing and reducing the symptoms of deficiency. Adopt the method of welcoming and replenishing and reducing. The treatment of "three needles in the pharynx" is a point-acupuncture method. In principle, no replenishing and reducing techniques can be performed after the point-acupuncture [3]. PD disease is located in the brain. Acupuncture on the brain acupoints of the Governor Meridian with “Tongdu Liyan” acupuncture can improve the patient's swallowing ability and at the same time improve the overall pathological state of the patient, achieving the purpose of treating the disease.

\section{The essentials of "Tongdu Liyan" acupuncture method}

During acupuncture, the patient was instructed in a semi-recumbent position. After routine disinfection, a disposable filiform needle with a size of $0.30 \times 40$ was selected for implementation. Yintang, Shenting, Baihui, and Naohu were all pierced 0.5-0.8 cun flatly, and Fengfu acupoint was inserted about 1 cun toward the tongue and pharynx. Among them, Yintang and Fengfu points were routinely pierced with the twisting method to replenish and relieve 1min. Shenting, Baihui, and Naohu points should be acupuncture based on syndrome differentiation. The patient's tongue coating and pulse condition suggests that the needle tip moves in the direction of the Governor Meridian during puncturing, and it prompts the positive patient to move the needle tip against the direction of the Governor Meridian; Lianquan point is inserted along the direction of the tongue base, and the direction of acupuncture at Fengfu point is in the same direction as the throat and directly to the disease. After that, Fengfu and Lianquan were connected to the electroacupuncture apparatus, and 2HZ continuous wave stimulation was selected. The needles of the above-mentioned points were kept for 30 minutes.

"three needles in the pharynx" [3]: The first needle is used to gently tap the back wall of the pharynx with a 1.5 cun needle, without bleeding; the second needle is used to tap the tip of the tongue with a 1.5 cun needle for slight bleeding or no bleeding; the third needle: Instruct the patient to roll the tongue and puncture the Jinjin and Yuye to cause slight bleeding (if the sublingual collaterals are severely tortuous, the area can be punctured at the same time).

\section{Contraindications of "Tongdu Liyan” acupuncture method}

Theoretically, the “Tongdu Liyan” acupuncture method can be used for all PD patients with swallowing disorders, but because PD itself may be accompanied by different degrees of mental disorders [6], such as anxiety, depression, hallucinations, delusions, etc., so do not use this acupuncture method for PD swallowing disorders with severe mental symptoms or other people who cannot cooperate, so as to avoid the danger of bending or breaking the needle. 


\section{Mechanism of action}

The swallowing process is divided into 4 phases, including the cognitive phase, oral phase, pharyngeal phase and esophageal phase. Swallowing disorders in PD patients are mainly in the first 3 phases. In the cognitive phase, patients usually cannot accurately identify the nature of food and cannot initiate swallowing actions. Oral and pharyngeal phases are usually due to muscle stiffness or poor coordination related to swallowing, resulting in slow eating, poor transportation, food residue, coughing and aspiration. The "Tongdu Liyan" acupuncture method mainly takes acupoints from the Du Channel, combined with the local acupoints of the diseased area to achieve a therapeutic effect. Its mechanism of action is introduced as follows:

\subsection{The basis of the role of TCM meridians}

\subsubsection{Governor Meridian and Brain}

The route of the Governor Meridian directly passes through the brain, and a branch of it originates from the inner canthus of the eye at the same time as the full sun bladder meridian. The genus of the Governor Meridian is also spreading. The qi of the six yang of the hands and feet is also injected into the head and face through the Governor Meridian acupoints.

The physiological function of the Governor Meridian is related to the brain. Traditional Chinese medicine teaches that the brain has a shape and an image, and pays more attention to its image, that is, the inner function and role of the brain. The brain occupies the position of the commander of the human body; "Medical Forest Correction": "The pure essence of the juice turns into the marrow and goes up from the spine into the brain". The most refined and pure things of the human body are finally transformed into the marrow and reach the brain. Then, the brain, as the residence of the essence, changes in its shape or image resulting in excess qi deficiency, strength and weakness, etc., will cause abnormal body functions, and even affect the original law of the body's growth and aging. The Governor Meridian passes upward through the Fengfu and Yamen to intersect with the Yang Wei Meridian in the brain. The Yangwei Meridian connects with the Yang Meridians throughout the body, restricts and regulates the accumulation of Qi and Blood in the Meridians, and makes them function within the normal range. The Governor Meridian is subordinate to the kidney, which is the innate foundation, and the main body is yin and yang. Enrichment of kidney qi can regulate the body and organs of the whole body. On the contrary, damage to the kidneys causes damage to the Governor Meridian, and damage to the Governor Meridian causes brain orifice disease.

\subsubsection{The Governor Meridian and Swallowing Disorder}

(1) The Governor Meridian follows the route of "going through the heart and entering the throat", and following the principle of "the meridian passes through, the main treatment is reached". The Governor Meridian has a therapeutic effect on the pathological changes of the throat. (2) The kidney meridian "follows the throat and pinches the tongue", and its meridian is tied to the root of the tongue. The "Huangdi Neijing Su Zhu" states that the jing point of kidney meridian has "anxious tongue, aphonia, throat discomfort". The manifestations and signs of "anxious tongue" and "throat discomfort" are similar to those of dysphagia. Then, due to the interaction between the Governor Meridian and the kidney meridian, stimulating the acupoints of the Governor Meridian can simultaneously clear the kidney meridian pathway and unblock the qi and blood in the pharynx, improve swallowing ability. The spleen meridian "holds the pharynx, connects the tongue, and spreads the tongue". Its meridian merges with the stomach meridian, and it binds upward in the throat and penetrates to the base of the tongue. After the liver meridian passes through the throat, it goes up into Hangsang, which refers to the throat and nasopharynx. It can be seen that the three meridians of the liver, spleen, and kidney can reach the tongue and pharynx in the circulation, and the Ren Meridian is the yin of any body, and it intersects with the Governor Meridian in the lower abdomen. It stimulates the Governor Meridian acupoints and can communicate with the Ren Meridian the imbalance of yin and yang, promoting the circulation of local qi and blood in the throat, and speeding up the process of swallowing. (3) Chong meridian, Ren meridian, and Governor meridian all originate from the first source of "Dan Tian", and they all pass through the throat and communicate with each other. If acupuncture is performed from the head points of the meridian, it can not only regulate the blood of the Ren meridian and Governor meridian at the junction of the throat, but also promote the completion of the swallowing action can also regulate the three meridians of Chong, Ren, and Governor, so as to promote the circulation of blood throughout the body of the patient, and improve the overall body condition of PD patients. About $10 \%$ of patients are diagnosed with PD with cognitive abnormality [7]. A large number of clinical and experimental studies have shown that the degree of cognitive impairment can be reduced by acupuncture at the Governor meridian acupoints [8]. Therefore, stimulation of the Governor Meridian can also improve the patient's cognitive ability to promote their recognition of food and shorten the time of the cogni- 
tive period of swallowing disorders.

\subsection{3 "Three needles in the pharynx" and dysphagia}

In "Lingshu·Genjie", it is pointed out that Jinjin and Yuye is the summary of the Qi in the upper part of the kidney meridian. "Leijing Tuyi" says: "Left Jinjin, right Yuye... Indications... swollen tongue, throat discomfort. Triangular needle bleeding" Swallowing disorders can be attributed to "throat discomfort”, "Tongdu Liyan” needle prick the tip of the tongue with three needles in the pharynx, Jinjin and Yuye can achieve the therapeutic effect.

\subsection{Western medicine mechanism of action}

\subsubsection{PD dysphagia and modern medical research}

Compared with the "brain" in traditional Chinese medicine, most of the current research in modern medicine tends to cause PD dysphagia due to damage to the substantia nigra of the midbrain, and the degeneration of the substantia nigra dense cells causes dopaminergic (DA) neuron apoptosis. Other studies have shown that the pathological changes of $\alpha$-synuclein are more closely related to the loss of substantia nigra neurons, which is a major cause of PD swallowing disorders [9]. Professor Braak divided Parkinson's disease into six stages and found that the pathological $\alpha$-synuclein in the first stage will invade the medulla oblongata (the basic center of swallowing), and the glossopharyngeal nerve and vagus nerve from the medulla oblongata will be implicated. Not only does it damage the main afferent pathway of the impulse to swallow, but also the muscle movement of the throat is weakened, and the epiglottis cannot be tilted back in time, which greatly increases the incidence of coughing or aspiration in patients. In the second stage, it mainly affects the autonomic nerve center. The abnormality of the parasympathetic nerve cannot guarantee the normal secretion of saliva. When the food consistency is not suitable for swallowing, it will affect the oral period of swallowing. In the third stage, $\alpha$-synaptic protein affects the substantia nigra of the midbrain, and the motor symptoms of PD gradually appear, and the patient's ability to live independently decreases. Therefore, the negative emotions that appear may also aggravate the dysphagia in the prodromal stage of PD. The end-stage sensory and motor cortex areas will be affected. This area is adjacent to the representative areas of the mouth, tongue, and throat of the head and face. The activation of the sensory area is not conducive to the introduction and summary of surrounding swallowing information [10]. Based on the close connection between the brain and PD dysphagia, the "Tongdu Liyan" acupuncture method selects the head points of the Governor Meridian to intervene, and the treatment is more targeted.

\subsubsection{Anatomical basis and experimental research}

The anatomical basis and experimental research of Governor Meridian acupoints are related to PD dysphagia. (1) Baihui acupoint is located in the suture between the two parietal bones, and there are abundant nerves and arteriovenous networks under it. Acupuncture Baihui acupoint can ensure that the brain, as a high-level center, has sufficient circulating blood volume to control the whole body's sensory activities. And some studies have pointed out that stimulating Baihui acupoint can increase and protect the DA neurons in the substantia nigra [11]. (2) Naohu acupoint is at the joint between the two parietal bones and the occipital bone [12]. The foramen magnum anterior and inferior to the occiput is the place where the brain migrates to the spinal cord. There is an outward opening of the hypoglossal nerve tube at the base of the occipital condyle. The method indirectly affects the nerve conduction and brain spinal cord function in this area, and better exerts its function of innervating tongue movement. (3) Fengfu acupoint is the swallowing center-the body surface projection of the medulla oblongata [13]. Intervention with this acupoint can more directly improve the control of the medulla oblongata on the swallowing process. At the same time, experiments have found that acupuncture at Fengfu acupoint can improve the substantia nigra of the midbrain DA cell survival rate, thereby protecting and promoting the repair of DA [14]. (4) Shenting, Yintang, and Baihui acupoints protect neurons in the hippocampus, improve cognitive ability [15-16], and improve cognitive impairment in patients with Parkinson's disease. (5) Lianquan acupoint and Fengfu acupoint relatively targeted the root of the tongue, the qi reaches the disease position, and the tongue and pharynx function recovers faster. (6) The intervention sites of "Three Needles in the Pharynx" all have nerves related to swallowing, such as the tongue nerve, hypoglossal nerve, vagus nerve, etc. Direct stimulation to rebuild its innervation function can better cooperate with the central nervous system to complete the swallowing process [5].

\section{Clinical case}

He Moumou, male, 70 years old, sees a doctor on August 9, 2020.

Main complaint: Slow action accompanied by drinking water and choking for half a year, worsening for 2 weeks. Medical history: Half a year ago, the patient gradually developed slowness of movement, difficulty in starting, and 
choking coughing while drinking water. He was diagnosed with "Parkinson's disease syndrome" at the Affiliated Hospital of Shaanxi University of Chinese Medicine and was given "Medobar" orally. The symptoms have been alleviated. In the past 2 weeks, the patient's conscious sluggishness has been aggravated compared with the previous period, and his walking dragged his steps. The family members found that his voice was hoarse. So he came to our hospital for further comprehensive diagnosis and treatment. Auxiliary examination: 1. Unified Parkinson Rating Scale (UPDRS): 70.5 points, in line with the diagnosis of Parkinson's disease; 2. Chinese version of the Dysphagia Questionnaire (SDQ-C): 26 points, suggesting that dysphagia is very likely; 3. Video fluoroscopy swallowing test (VFSS): swallowing dysfunction when empty swallowing, delayed swallowing start, multiple swallows; prolonged tongue movement time when drinking water, indicating weak tongue muscle motility and slow swallowing start; 4. Random blood glucose: 9.0mmol/L. Tongue status: dark red tongue with little coating; pulse status: narrow pulse. Specialty status: Consciousness, poor spirit, mask face, slight decline in orientation, memory, calculation, comprehension, and judgment; lack of fluency in speech, no uselessness, and ignorance. The frontal lines are symmetrical on both sides, the eyes are closed normally, and the nasolabial folds are symmetrical on both sides. The uvula is centered, and the bilateral pharyngeal reflex is slightly weakened. The tongue extension is in the middle, the tongue muscle strength is normal, and the tongue muscle has no wretchedness and tremor. Extremities muscle strength is level 5, extremities muscle tension is slightly higher, and tendon reflexes are symmetrical. There is no abnormality in bilateral deep and shallow sensation, pathological reflex is not elicited, and meningeal irritation signs are negative.

Chinese medicine diagnosis: tremor syndrome (liver and kidney deficiency syndrome), western medicine diagnosis: 1. Parkinson's disease syndrome 2. Swallowing dysfunction. Governance: tonic liver and kidney, nourish muscles and veins. Treatment: (1) Western medicine supports symptomatic treatment with anti-tremor paralysis, lipid-regulating and stabilizing spots, improving cerebral blood supply, nutrient cells, etc.; (2) "Tongdu Liyan" acupuncture method, acupuncture prescription: main point: pharynx three needles, Lianquan, Shenting, Yintang, Baihui, Fengfu, Naohu. Acupoint selection based on syndrome differentiation: Taichong and Taixi. Operation: The patient takes the supine position, $75 \%$ alcohol routinely disinfects the acupuncture site, and takes a $0.30 \times 40$ disposable acupuncture needle. The needle is inserted 1 cun from the Fengfu point toward the throat, and the Shenting, Baihui, and Naohushundu channels are punctured flatly. Approximately 0.8 cun, Yintang and Lianquan routine acupuncture, the above acupoints were flattened and relieved for $1 \mathrm{~min}$; then a 1.5 inch needle was used to puncture the throat wall, tongue tip and Jinjin and Yuye without any manipulation. Then choose Fengfu and Lianquan to connect to the electroacupuncture device and stimulate with $2 \mathrm{HZ}$ continuous wave. The treatment is once a day, 30 minutes each time, 6 times a week, a total of 1 month of treatment.

At the second clinic on September 16, 2020, the patient complained of slowness of movement, difficulty in starting, and coughing when drinking water. The patient had not complained of special discomfort. Scale evaluation: 1. UPDRS: 65 points, 2. SDQ-C: 23 points. Acupuncture treatment was maintained in the outpatient clinic for 2 months, during which the condition was stable. He was instructed to take active facial and oral function exercises, take medicines on time, be emotional, and follow up if he feels unwell.

Note: The patient is old and weak, insufficient kidney essence, coupled with chronic illness and disorder, draining the yin of the liver and kidney, deficiency of the yin of the liver and kidney, unable to reach the throat and tongue, losing nourishing, and swallowing difficult. At this time, the deficiency is the main body. During the treatment, Mr. Feng uses conventional drugs to cooperate with the "Tongdu Liyan" acupuncture method. Acupuncture is suitable for supplementation, and Taichong and Taixi are used to nourish liver and kidney, disperse liver qi, and harvest good results.

\section{Conclusion}

Dysphagia can occur at any stage of PD, even throughout [17]; damage to the brain structure and nervous system in different clinical stages of PD will also affect the swallowing ability of patients at all levels, so early intervention is particularly important. In summary, the "Tongdu Liyan" acupuncture method treatment for PD dysphagia is a summary of the author's instructor's experience in clinical practice. Its effectiveness has been demonstrated clinically and scientifically, and it is worth continuing to study to benefit patients.

\section{References}

[1] Kyung Duck Lee, Jung Hoi Koo, Sun Hong Song, et al. (2015). Central cholinergic dysfunction could be associated with oropharyngeal dysphagia in early Parkinson's disease [J]. Journal of neural transmission (Vienna, Austria: 1996), 2015,122 (11): 1553-1561. 
[2] Chen Miao, Huang Jianping. (2020). Research progress in rehabilitation and traditional Chinese medicine treatment of Parkinson's disease with dysphagia [J]. Journal of Neurology and Neurorehabilitation, 2020, 16(03): 121-128.

[3] Feng Weixing. (2012). Three pharynx needles combined with electrical stimulation to treat 68 cases of dysphagia after stroke [J]. Journal of Acupuncture and Moxibustion, 2012, 28(07): 16-17.

[4] Feng Weixing, Li Pei, Yan Yongmei, et al. (2015). Acupuncture treatment of 45 cases of dysphagia after stroke [J]. Shaanxi Journal of Traditional Chinese Medicine, 2015, 36(01): 91-93.

[5] Wang Xiao, Feng Weixing, Tian Shen, et al. (2020). Treatment of Parkinson's disease swallowing dysphagia with three pharyngeal acupuncture and eight anti-tremor acupuncture [J]. Jilin Journal of Traditional Chinese Medicine, 2020, 40(10): 1383-1386.

[6] Li Huiying, Li Yanfeng. (2019). Parkinson's disease-related mood disorders [J]. Chinese Journal of Neuroimmunology and Neurology, 2019, 26(06): 454-459.

[7] Zhang Feng, Che Ningning, Li Xue, et al. (2020). Analysis of the correlation and influencing factors between leukoaraiosis and cognitive impairment in patients with Parkinson's disease [J]. Chinese Journal of Practical Diagnosis and Therapy, 2020, 34(09): 932-935.

[8] Zheyan Sa, Qianru Huang, Xiaohua Pan, et al. (2018). The effect of electric acupuncture on the infrared temperature of the Governor Vessel in cognitively impaired rats [J]. Modern Distance Education of Chinese Medicine, 2018, 16(17): 88-91.

[9] Yang Zhao, Kang Wenyan, Liu Jun. (2020). The correlation study of salivary DJ-1 protein with non-motor symptoms and $\alpha$-synuclein in patients with primary Parkinson's disease [J]. Journal of Clinical Internal Medicine, 2020, 37 (06): $423-426$.

[10] Leng Fangda, Wang Yawei, Liu Fang, et al. (2020). Pathogenesis of swallowing dysfunction and progress in rehabilitation in patients with Parkinson's disease [J]. Medical Review, 2020, 26(17): 3451-3455.

[11] Li Xia, Li Jing, Wang Yongfeng, et al. (2014). Effect of electroacupuncture on behavior and midbrain substantia TH in Parkinson's rats [J]. Hebei Medicine, 2014, 36(04): 506-509.

[12] Liu Li'an, Meng Yue, Wang Yulin, et al. (2019). Exploration of Suihai Theory and Application of Naohu Acupoints [J]. Chinese Acupuncture and Moxibustion, 2019, 39(09): 1003-1006.

[13] Zhang Haidong, Yu Ansheng, Li Fengmei, et al. (2003). Three-dimensional reconstruction of the anatomical structure of Fengfu point [J]. Acupuncture Research, 2003, (01): 58-61.

[14] Ma Jun, Wang Yao, Wang Shuju, et al. (2019). Regulation of EIF2 $\alpha$-ATF4-GRP78 pathway in Parkinson's disease rats by electro-acupuncture at “Fengfu” and “Taichong” points [J]. Acupuncture Research, 2019, 44(12): 873-877.

[15] Zhang Yiyuan, Du Yuanhao. (2020). Preliminary study on the prescription of acupuncture and moxibustion for mild cognitive impairment [J]. Tianjin Traditional Chinese Medicine, 2020, 37(01): 65-70.

[16] Zhan Feiyan, Wei Jiaohua, Wu Renqing. (2020). The effect of Baihui and Yintang points on the cognitive function of rats after sevoflurane anesthesia and its mechanism [J]. World Chinese Medicine, 2020, 15(05): 738-742.

[17] Wang Hongyan, Zuo Guanchao, Liu Jing, et al. (2020). Observation on the efficacy of acupuncture combined with oral sensorimotor training in the treatment of Parkinson's disease dysphagia [J]. Shanghai Journal of Acupuncture, 2020, 39(04): 440-445. 